\title{
Improving hole injection efficiency by manipulating the hole transport mechanism through $p$-type electron blocking layer engineering
}

\author{
Zi-Hui Zhang, ${ }^{1}$ Zhengang Ju, ${ }^{1,+}$ Wei Liu, ${ }^{1}$ Swee Tiam Tan, ${ }^{1}$ Yun Ji, ${ }^{1}$ Zabu Kyaw, ${ }^{1}$ Xueliang Zhang, \\ Namig Hasanov, ${ }^{1}$ Xiao Wei Sun, ${ }^{1}$ and Hilmi Volkan Demir ${ }^{1,2, *}$ \\ ${ }^{1}$ LUMINOUS! Centre of Excellence for Semiconductor Lighting and Displays, School of Electrical and Electronic Engineering, \\ School of Physical and Mathematical Sciences Nanyang Technological University, 50 Nanyang Avenue, Singapore 639798 \\ ${ }^{2}$ Department of Electrical and Electronics, Department of Physics, and UNAM-Institute of Material Science and Nanotechnology, \\ Bilkent University, TR-06800 Ankara, Turkey \\ *Corresponding author: volkan@stanfordalumni.org
}

Received January 14, 2014; accepted March 11, 2014;

posted March 17, 2014 (Doc. ID 204641); published April 14, 2014

\begin{abstract}
The $p$-type AlGaN electron blocking layer (EBL) is widely used in InGaN/GaN light-emitting diodes (LEDs) for electron overflow suppression. However, a typical EBL also reduces the hole injection efficiency, because holes have to climb over the energy barrier generated at the $p$-AlGaN $/ p$-GaN interface before entering the quantum wells. In this work, to address this problem, we report the enhancement of hole injection efficiency by manipulating the hole transport mechanism through insertion of a thin GaN layer of $1 \mathrm{~nm}$ into the $p$-AlGaN EBL and propose an $\mathrm{AlGaN} / \mathrm{GaN} / \mathrm{AlGaN}$-type EBL outperforming conventional AlGaN EBLs. Here, the position of the inserted thin GaN layer relative to the $p$-GaN region is found to be the key to enhancing the hole injection efficiency. InGaN/ GaN LEDs with the proposed $p$-type AlGaN/GaN/AlGaN EBL have demonstrated substantially higher optical output power and external quantum efficiency. (c) 2014 Optical Society of America

OCIS codes: (230.3670) Light-emitting diodes; (230.5590) Quantum-well, -wire and -dot devices; (160.6000) Semiconductor materials.

http://dx.doi.org/10.1364/OL.39.002483
\end{abstract}

Regarded as the ultimate light sources, InGaN/GaN light-emitting diodes (LEDs) have gained intensive interest, and tremendous progress has been made on the development of LEDs in the last several decades [1]. However, the device performance is still limited by a low hole injection efficiency into the InGaN/ GaN multiple quantum wells (MQWs). Thus, significant efforts have been made to engineer the quantum barriers to enhance the hole transport [2,3]. Meanwhile, in the commonly adopted $p$-type AlGaN electron blocking layer (EBL), a hole-blocking effect by the $p$-type EBL has been observed and reported [4]. The consequence of the hole-blocking effect is the electron leakage from the InGaN/GaN MQW region and the lower hole concentration taking part in the radiative recombination [5]. To address this issue, different types of EBLs have been proposed to enhance the hole injection efficiency [ $\underline{6}-\underline{8}]$. However, all these proposed EBL structures can, on one hand, reduce the barrier height between the $p$-type EBL and the $p$-GaN layer for hole transport promotion, while the barrier height for electrons is, on the other hand, also simultaneously reduced, thus leading to a reduced blocking effect on the electrons leaking from the InGaN/GaN MQW region. Most recently, Xia et al. have theoretically demonstrated a $p$-type AlGaN/GaN/AlGaN EBL with a GaN intermediate layer thicker than $4 \mathrm{~nm}$ [9], which promises to both enhance the electron blocking and promote the hole transport. Although the optical power and the external quantum efficiency (EQE) have been predicted to be enhanced with the p-type AlGaN/GaN/ AlGaN EBL, the experimental confirmation of the effectiveness of the $p$-type AlGaN/GaN/AlGaN EBL has not been reported yet to date.
In this work we propose a $p$-type AlGaN/GaN/AlGaN EBL with a very thin GaN layer of $1 \mathrm{~nm}$ inserted in the $p$-AlGaN EBL and study its effect both theoretically and experimentally on the carrier transport in InGaN/ GaN LEDs. It is found in our theoretical analysis that, unlike the thicker GaN insertion layers (from 4 to $16 \mathrm{~nm}$ ) in Xia et al.'s work, the newly formed quantum states in the GaN thin layer play a critical role in the hole transport promotion. Furthermore, the hole injection efficiency is also found to be sensitive to the position of the GaN thin layer relative to the $p$-GaN region. More importantly, our proposed EBL improves the carrier injection efficiency by increasing both the thermionic emission and tunneling processes for the holes $[10,11]$. These findings have been experimentally verified through the performance improvement of InGaN/GaN LEDs with the $p$-type AlGaN/GaN/AlGaN EBLs.

Three InGaN/GaN LED epitaxial wafers as shown in Fig. 1 were grown by a metal-organic chemical vapor deposition system. The growth was initiated from a $c$-plane sapphire substrate. We grew a $30 \mathrm{~nm}$ thick low-temperature GaN nucleation layer before growing

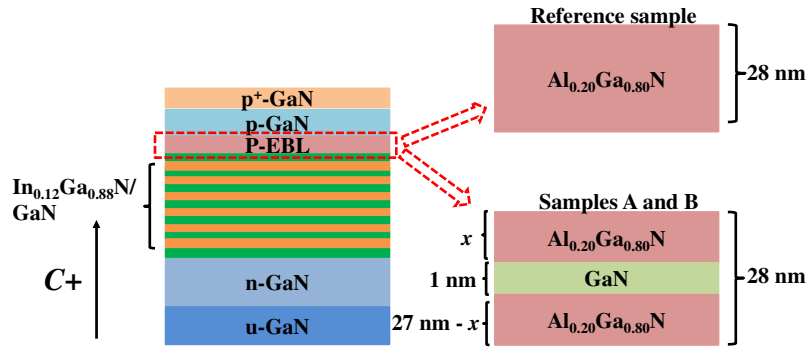

Fig. 1. Schematic diagrams for the reference sample, Sample A $(x=13.5 \mathrm{~nm})$, and Sample B $(x=3.5 \mathrm{~nm})$. 
the $4 \mu \mathrm{m}$ high-temperature unintentionally doped GaN $(u$-GaN) template. The following $n$-GaN layer of $2 \mu \mathrm{m}$ was doped with Si dopants, and the Si doping concentration was estimated to be $5 \times 10^{18} \mathrm{~cm}^{-3}$. All the LED samples have six pairs of $\operatorname{In}_{0.12} \mathrm{Ga}_{0.88} \mathrm{~N} / \mathrm{GaN}$ MQWs, and the thicknesses of the quantum wells and quantum barriers are 3 and $12 \mathrm{~nm}$, respectively. For the reference sample, a $28 \mathrm{~nm}$ thick $p$-type $\mathrm{Al}_{0.20} \mathrm{Ga}_{0.80} \mathrm{~N}$ EBL was grown, and the hole concentration was estimated to be $1 \times 10^{17} \mathrm{~cm}^{-3}$. In contrast, a $p-\mathrm{Al}_{0.20} \mathrm{Ga}_{0.80} \mathrm{~N} / u$-GaN $/ p-\mathrm{Al}_{0.20} \mathrm{Ga}_{0.80} \mathrm{~N}$ EBL was grown for Samples A and $\mathrm{B}$, of which the effective hole concentration of the $p-\mathrm{Al}_{0.20} \mathrm{Ga}_{0.80} \mathrm{~N}$ regions was also $1 \times 10^{17} \mathrm{~cm}^{-3}$, while the GaN region was undoped. The thickness of the embedded thin GaN layer was controlled to be $1 \mathrm{~nm}$ by the growth time, during which the TMAl and $\mathrm{Cp}_{2} \mathrm{Mg}$ supplies were cut off. The growth temperature of the EBL region for both LED epitaxial wafers was kept to $973^{\circ} \mathrm{C}$ to protect the quantum wells. In addition, the growth pressure was set to 100 mbars. In addition, as shown in Fig. 1, the position of the thin GaN layer was varied in Samples A and $\mathrm{B}$. We define $x$ as the thickness of the remaining $p-\mathrm{Al}_{0.20} \mathrm{Ga}_{0.80} \mathrm{~N}$ EBL between the thin $u$-GaN and the $p$-GaN region, and it was set to 13.5 and $3.5 \mathrm{~nm}$ for Samples A and B, respectively. Finally, all the LED samples were covered by a $0.2 \mu \mathrm{m}$ thick $p$-GaN layer, and the effective hole concentration was $3 \times 10^{17} \mathrm{~cm}^{-3}$. Those $p$-type layers were finally in situ annealed for $600 \mathrm{~s}$ under $730^{\circ} \mathrm{C}$ in the $\mathrm{N}_{2}$ ambient.

The electroluminescence (EL) spectra and the optical output power for the three LED samples were measured through a calibrated integrating sphere attached to a calibrated Ocean Optics spectrometer (QE65000). The measurements were conducted on the LED dies with a diameter of $1.0 \mathrm{~mm}$.

The EL spectra collected at a current injection level of $30 \mathrm{~A} / \mathrm{cm}^{2}$ for the reference sample, Sample A, and Sample B are shown in Fig. 2. It is shown that Samples $\mathrm{A}$ and $\mathrm{B}$ emit stronger EL intensities than the reference sample with Sample B being the strongest optically among all. Besides, the peak emission wavelengths are observed to be 434, 437 and $431 \mathrm{~nm}$ for the Reference sample, Sample A, and Sample B, respectively. We attribute the small emission wavelength variation to the growth fluctuations.

The optical output power and EQE are presented in Fig. 3. Being consistent with Fig. 2 , the reference sample shows the lowest in the optical output power and the

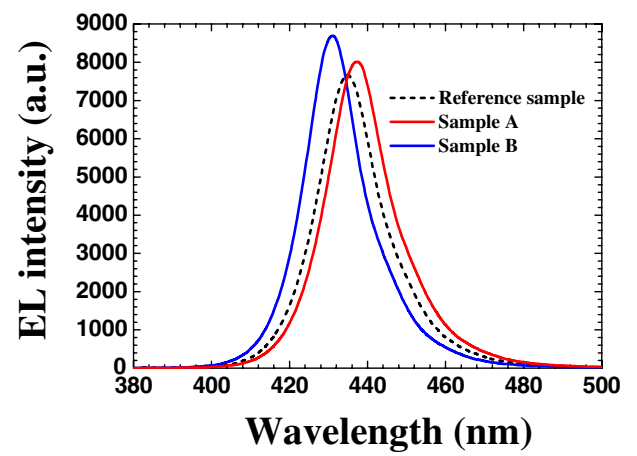

Fig. 2. EL spectra from the reference sample, Sample A, and Sample B at $30 \mathrm{~A} / \mathrm{cm}^{2}$.

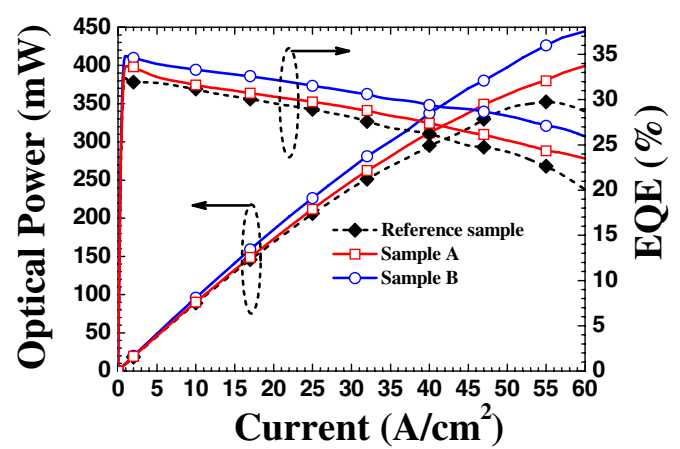

Fig. 3. Optical output power and EQE for the reference sample, Sample A, and Sample B.

EQE. The device performance is improved in Sample A, while the largest enhancement is obtained from Sample B. For example, the power enhancement for Sample A and Samples B is $3.3 \%$ and $9.6 \%$ at $30 \mathrm{~A} / \mathrm{cm}^{2}$, respectively, when compared to the reference sample. We have attributed the enhanced power for Samples A and B to the increased hole injection into the InGaN/GaN MQW region, and the details on the hole injection enhancement will be discussed subsequently.

To clarify the origin of the device performance improvement achieved above, we have simulated the valence band structure, the hole concentration profiles, and the radiative recombination rates for the reference, Sample A, and Sample B. The simulations have been conducted by APSYS [12], which self-consistently solves the Poisson equations and the Schrödinger equation with proper boundary conditions. The carrier transport model takes the carrier drift and diffusion into consideration. Specifically, besides employing the thermionic emission process for carriers to transport across any heterointerfaces, such as the InGaN/GaN MQW region, the $p$-type $\mathrm{EBL}$, and the $p$-GaN layer, we also consider the thermally assisted tunneling process by treating the $\mathrm{AlGaN}$ region as a rectangular energy barrier [5]. We also take into account the Auger recombination and the ShockleyRead-Hall recombination within the InGaN/GaN MQW region for carrier loss. Furthermore, we include the piezoelectric and spontaneous polarization effects in heterointerfaces in our models. The detailed material parameters used in our simulations can be found in our previously published works $[3,13,14]$.

To investigate the hole transport mechanism, we have calculated the valence band diagrams for the samples with the conventional bulk $p$-AlGaN EBL and the $p$-AlGaN $/ u$-GaN $/ p$-AlGaN EBL shown in Figs. 4(a) and $\underline{4(b)}$, respectively. The hole current, which is injected into the $p$-type AlGaN EBL through the thermionic emission process in Fig. 4(a), can be expressed by $J_{\mathrm{GaN} \rightarrow \mathrm{AlGaN}} \sim \exp \left(-\Phi_{b} / k T\right)$, while $\Phi_{b}=\Delta E_{V}-k T \times$ $\ln \left(p_{p \text {-GaN }} / N_{V}\right)[\underline{15}]$ and $\Delta E_{V}=E_{V_{-} \mathrm{GaN}}-E_{V_{-} \mathrm{AlGaN}}$, where $E_{V_{-} \text {GaN }}$ and $E_{V \_ \text {AlGaN }}$ are the valence band edges for GaN and AlGaN, respectively, $N_{V}$ is the effective density of states for holes, $p_{p \text {-GaN }}$ is the nonequilibrium hole concentration in the $p_{p-\text { GaN }}$ region, $k$ is the Boltzmann constant, and $\Phi_{b}$ is the barrier height for holes. Clearly we can see that one can reduce $\Phi_{b}$ by reducing the valence band offset $\Delta E_{V}$ between the $p$-AlGaN and the $p$-GaN. One 
approach to reduce $\Delta E_{V}$ is to reduce the AlN composition in the AlGaN layer. However, reducing the AlN composition in the AlGaN layer results in an increased electron overflow out of the EBL. Another way is to increase the hole concentration by heavily doping the $p$-type layers. However, the $p$-type doping efficiency is low for both GaN and AlGaN compounds [16], which makes a high $p_{p \text {-GaN }}$ less probable. Nevertheless, the barrier height for holes can be modified by inserting a thin GaN layer in the $p$-AlGaN region, as shown in Fig. 4(b). Due to the small thickness of the inserted $u$ GaN layer, a series of quantum states are formed in the $u$-GaN layer. Therefore, in addition to the direct thermionic emission process for the high-energy holes to be injected into the EBL by overcoming $\Phi_{b}$, those holes with energy smaller than $\Phi_{b}$ can tunnel into the quantum states and then are injected into the InGaN/GaN MQW region by climbing over the next barrier height of $\emptyset_{b}$, where $\quad \emptyset_{b}=\Delta E_{V i}-k T \times \ln \left(p_{\mathrm{AlGaN} / \mathrm{GaN} / \mathrm{AlGaN}} / N_{V}\right) \quad$ with $\Delta E_{V i}=E_{V i_{-} \mathrm{GaN}}-E_{V \_ \text {AlGaN }}$. Here $E_{V i_{-} \mathrm{GaN}}$ is the quantized subvalance band edge of the thin GaN layer in the $p$-AlGaN/ $u$-GaN $/ p$-AlGaN EBL, and $p_{\mathrm{AlGaN} / \mathrm{GaN} / \mathrm{AlGaN}}$ is the hole concentration in the quantized subvalance band for the $p$-AlGaN $/ u$-GaN $/ p$-AlGaN well region. The current contributed by the holes that first tunnel into the quantum states and then are injected into the InGaN/ GaN MQW region through thermionic emission can be described as $J_{\mathrm{GaN} \rightarrow \mathrm{AlGaN}} \sim \exp \left(-\varnothing_{b} / k T\right)$. Since $E_{V i_{-} \mathrm{GaN}}$ is smaller than $E_{V_{-} G a N}, \Delta E_{V i}$ is smaller than $\Delta E_{V}$. In addition, the $u$-GaN region in the $p$-AlGaN $/ u$-GaN $/ p$-AlGaN EBL is so thin that the electrons are less likely to be confined in it, which avoids hole loss through radiative recombination with the nonequilibrium electrons in the $u$-GaN layer. Thereby the quasi-Fermi level (i.e., $E_{f h}$ ) in the $p$-AlGaN $/ u$-GaN $/ p$-AlGaN-type EBL region is flat, as shown in Fig. 4(b). Also, the holes that have occupied the quantized energy states are strongly confined by the AlGaN layer, making the hole concentration in the thin $u$-GaN layer $p_{\mathrm{AlGaN} / \mathrm{GaN} / \mathrm{AlGaN}}$ much higher than that in the $p$-GaN region. Therefore $\emptyset_{b}$ is much smaller than $\Phi_{b}$, as depicted in Fig. 4(b), which guarantees that those holes with energy between $\varnothing_{b}$ and $\Phi_{b}$ can be injected into the InGaN/GaN MQW region. Hence, the overall injection efficiency for holes can be enhanced, which accounts for the enhanced optical output power and EQE for Samples $\mathrm{A}$ and B when compared to the reference sample.

The even better performance of Sample B than Sample A indicates that the relative position of the thin $u$-GaN layer to the p-GaN region $x$ is key to further enhancing the hole transport. Obviously the smaller the value of $x$,
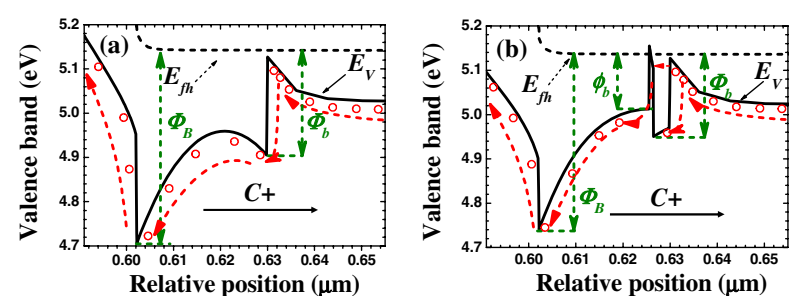

Fig. 4. Hole transport mechanism for (a) the sample with a bulk $p$-type EBL and (b) the proposed sample with a $p$-AlGaN $/ u$-GaN $/ p$-AlGaN-type EBL at $30 \mathrm{~A} / \mathrm{cm}^{2} . E_{V}$ denotes the valence band, and $E_{f h}$ is the quasi-Fermi level for holes. the higher the hole-tunneling efficiency [15]. Therefore, Sample B has a larger hole-tunneling efficiency than Sample A, which explains the larger optical output power and EQE for Sample B than those for Sample A.

The influence of the insertion of the thin $u$-GaN layer in the $p$-AlGaN $/ u$-GaN $/ p$-AlGaN-type EBL and the relative position of the $u$-GaN layer on the energy barrier heights for holes are summarized in Table 1 . Samples A and B exhibit a smaller $\Phi_{b}$ than the reference sample, which indicates that the $\Phi_{b}$ can be reduced by employing a $p$-AlGaN $/ u$-GaN $/ p$-AlGaN-type EBL. Meanwhile, given the polarization-induced electric field within the AlGaN layer for the AlGaN/GaN/AlGaN epitaxial architecture with a periodic boundary [17], we know that the barrier height caused by the polarization-induced electric field can be approximately expressed by $\Phi_{b}=e \times \sigma_{\mathrm{pol}} \times$ $l_{\mathrm{GaN}} /\left(\varepsilon_{\mathrm{GaN}}+\varepsilon_{\mathrm{AlGaN}} \times l_{\mathrm{GaN}} / x\right)-e \times V_{\mathrm{ext}}$, where $l_{\mathrm{GaN}}=1 \mathrm{~nm}$, $\sigma_{\mathrm{pol}}$ is the polarization-induced interface charge density between $\mathrm{GaN}$ and AlGaN layers, $e$ is the elementary electronic charge, and $\varepsilon_{\mathrm{GaN}}$ and $\varepsilon_{\mathrm{AlGaN}}$ are the dielectric constants for GaN and AlGaN, respectively. $V_{\text {ext }}$ is the voltage drop across the AlGaN region with a thickness of $x$. Since Sample B has a smaller value of $x$ than Sample A, Sample B possesses an even smaller $\Phi_{b}$ than Sample A, and this suggests an enhanced hole transport efficiency (in our model, we have considered both the thermionic emission and thermally assisted tunneling process for any heterointerfaces). Therefore, a high hole concentration in the thin $u$-GaN region of Sample B promises a reduced $\varnothing_{b}$ at the steady state. Furthermore, a smaller $\emptyset_{b}$ for Sample B than for Sample A demonstrates a higher hole escape efficiency from the thin $u$-GaN layer embedded in the $p$-AlGaN $/ u$-GaN $/ p$-AlGaN-type EBL, which on the other hand proves the excellent hole transport process through the thinner AlGaN layer in Sample B. Once the holes arrive at the EBL, their injection into the InGaN/GaN MQW region is influenced by $\Phi_{B}$, as shown in Figs. 4(a) and 4(b). In Table 1, Samples A and $\mathrm{B}$ have presented a reduced $\Phi_{B}$ compared to the reference sample, while Sample B shows the lowest $\Phi_{B}$.

Figure 5(a) shows the hole concentration in the InGaN/ GaN MQW regions for the three LED samples. As has been discussed previously, Samples A and B enable a higher hole injection efficiency than the reference, with Sample B being the highest, and correspondingly, the hole concentration in the InGaN/GaN MQW region for the reference is the smallest among the three samples. Meanwhile, Sample B has exhibited an even higher hole concentration across the MQW region than Sample A. The radiative recombination rates across the MQW regions for the studied samples are shown in Fig. 5(b). Consistent with Fig. 5(a), Sample B shows the most enhanced radiative recombination levels among the

Table 1. Energy Barrier Heights for the Reference, Sample $A$, and Sample $B$ at an Injection Current Level of $30 \mathrm{~A} / \mathrm{cm}^{2}$

\begin{tabular}{lccc}
\hline & $\phi_{b}(\mathrm{meV})$ & $\Phi_{b}(\mathrm{meV})$ & $\Phi_{B}(\mathrm{meV})$ \\
\hline Reference sample & & 246.0 & 445.4 \\
Sample A & 183.8 & 239.8 & 427.9 \\
Sample B & 143.8 & 206.1 & 415.3 \\
\hline
\end{tabular}



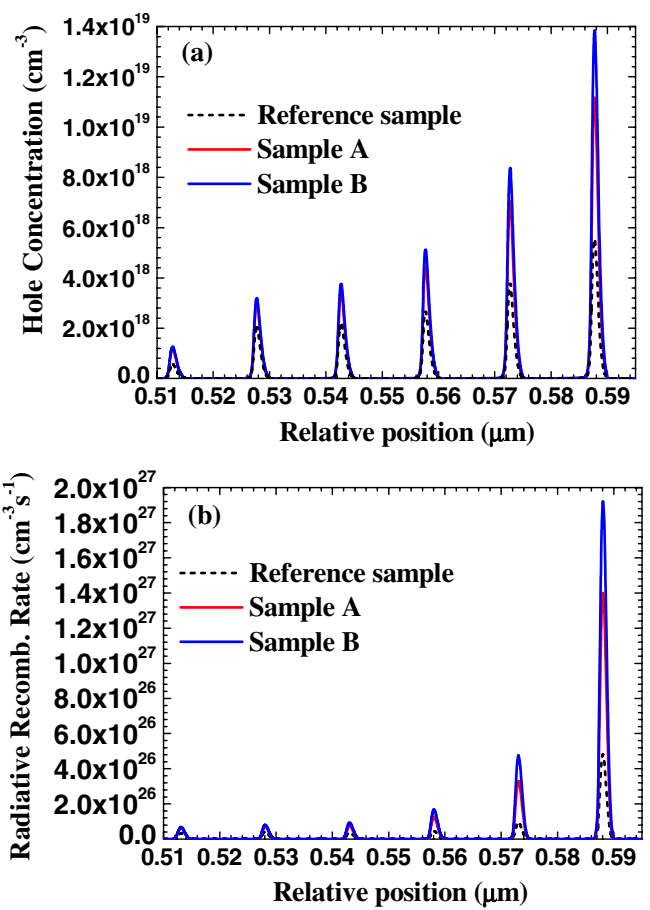

Fig. 5. Simulated (a) hole distributions and (b) radiative recombination rates in the MQW region at $30 \mathrm{~A} / \mathrm{cm}^{2}$ for the reference, Sample A, and Sample B.

three samples. The strongest radiative recombination rate for Sample B is due to the most improved hole transport, which is the result of the combined effect of the thin $u$-GaN layer inserted in the $p$-AlGaN $/ u$-GaN $/ p$-AlGaN EBL and the strong hole tunneling taking place through the thin remaining AlGaN layer.

To summarize, InGaN/GaN LEDs with a $p$-AlGaN/ $u$-GaN/p-AlGaN-type EBL have been studied. Compared to the conventional $p$-type bulk AlGaN EBL, the proposed structure features an increased hole injection, and thus an enhanced optical output power and EQE among the proposed samples. In addition, in order to eliminate the hole loss due to recombining with electrons, the GaN layer has to be thin in the $p$-AlGaN $/ u$-GaN $/ p$-AlGaN EBL. Also, we have found that the position of the thin GaN in the $p$-AlGaN/ $u$-GaN/ $p$-AlGaN EBL is very sensitive to the hole injection, and a GaN region close to the $p$-GaN layer is preferable for a better hole injection. We believe the $p$-AlGaN/ $u$-GaN/p-AlGaN-type electron-blocking layer is promising for high-performance InGaN/GaN LEDs and will inspire variants of such EBL designs.

This work is supported by the National Research Foundation of Singapore under Grant No. NRF-CRP-6-2010-2 and NRF-RF-2009-09 and the Singapore Agency for Science, Technology and Research (A*STAR) SERC under Grant No. 1121202009.

$\dagger$ Equal contribution to first author

\section{References}

1. S. T. Tan, X. W. Sun, H. V. Demir, and S. P. DenBaars, IEEE Photon. J. 4, 613 (2012).

2. Z.-H. Zhang, S. T. Tan, Y. Ji, W. Liu, Z. Ju, Z. Kyaw, X. W. Sun, and H. V. Demir, Opt. Express 21, 15676 (2013).

3. Z. G. Ju, W. Liu, Z. H. Zhang, S. T. Tan, Y. Ji, Z. B. Kyaw, X. L. Zhang, S. P. Lu, Y. P. Zhang, B. B. Zhu, N. Hasanov, X. W. Sun, and H. V. Demir, Appl. Phys. Lett. 102, 243504 (2013).

4. S.-H. Han, D.-Y. Lee, S.-J. Lee, C.-Y. Cho, M.-K. Kwon, S. P. Lee, D. Y. Noh, D.-J. Kim, Y. C. Kim, and S.-J. Park, Appl. Phys. Lett. 94, 231123 (2009).

5. J. Piprek and Z. M. Simon Li, Appl. Phys. Lett. 102, 023510 (2013).

6. Y.-K. Kuo, J.-Y. Chang, and M.-C. Tsai, Opt. Lett. 35, 3285 (2010).

7. Y.-H. Lu, Y.-K. Fu, S.-J. Huang, Y.-K. Su, R. Xuan, and M. H. Pilkuhn, Appl. Phys. Lett. 102, 143504 (2013).

8. Y. Y. Zhang and Y. A. Yin, Appl. Phys. Lett. 99, 221103 (2011).

9. C. S. Xia, Z. M. S. Li, W. Lu, Z. H. Zhang, Y. Sheng, W. Da Hu, and L. W. Cheng, J. Appl. Phys. 111, 094503 (2012).

10. C. H. Wang, C. C. Ke, C. Y. Lee, S. P. Chang, W. T. Chang, J. C. Li, Z. Y. Li, H. C. Yang, H. C. Kuo, T. C. Lu, and S. C. Wang, Appl. Phys. Lett. 97, 261103 (2010).

11. Y.-Y. Lin, R. W. Chuang, S.-J. Chang, S. Li, Z.-Y. Jiao, T. Ko, S. J. Hon, and C. H. Liu, IEEE Photon. Technol. Lett. 24, 1600 (2012).

12. Z.-H. Zhang, S. T. Tan, Z. Ju, W. Liu, Y. Ji, Z. Kyaw, Y. Dikme, X. W. Sun, and H. V. Demir, J. Display Technol. 9, 226 (2013).

13. Z.-H. Zhang, S. T. Tan, W. Liu, Z. Ju, K. Zheng, Z. Kyaw, Y. Ji, N. Hasanov, X. W. Sun, and H. V. Demir, Opt. Express 21, 4958 (2013).

14. Z.-H. Zhang, S. Tiam Tan, Z. Kyaw, Y. Ji, W. Liu, Z. Ju, N. Hasanov, X. W. Sun, and H. V. Demir, Appl. Phys. Lett. 102, 193508 (2013).

15. S. M. Sze, Physics of Semiconductor Devices, 2nd ed. (Wiley, 1981).

16. T. Tanaka, A. Watanabe, H. Amano, Y. Kobayashi, I. Akasaki, S. Yamazaki, and M. Koike, Appl. Phys. Lett. 65, 593 (1994).

17. J. Piprek, Nitride Semiconductor Devices: Principles and Simulation (Wiley-VCH, 2007). 